\title{
POSITIONING SYSTEM OF A PNEUMATIC ACTUATOR DRIVEN BY PROPORTIONAL PRESSURE REGULATOR VALVES
}

\author{
Gabriel Almeida Muzy \\ Emerson Industrial Automation FLMC \\ Barueri, São Paulo, Brazil
}

\author{
Alexandre Simião Caporali \\ Federal Institute of Education, Science and \\ Technology of São Paulo (IFSP) \\ São Paulo, São Paulo, Brazil
}

\begin{abstract}
This paper presents a positioning system of a linear double acting pneumatic cylinder commanded by two proportional pressure regulator valves. The advantages presented by pneumatic systems make this technology reason for constant research in the scientific field. But, the nonlinearities related to the use of compressed air and the involved frictions in the movement make the control complex. In many researches about this subject, the authors adopted proportional valves to control the air mass flow directed to the rear and front chambers of the pneumatic cylinder. In the present research, the proportional valves controlled the air pressure instead of air mass flow. The pneumatic cylinder was equipped with an internal linear resistive transducer and the proportional pressure regulator valves were set to work in double loop mode, monitoring and controlling the input and output signals of the process. Each valve was equipped with an onboard proportional integral (PI) controller which were tuned according to the second technique proposed by Ziegler-Nichols. To make possible the execution of simulations, mathematical models were developed taking into account the piston dynamics, the compressed air behavior inside the front and rear chambers, the static and dynamic frictions and the valves dynamics. To validate the mathematical model proposed, comparisons between the experimental data, collected from a prototype connected to the software Labview, and the computational simulations results, that were performed in the software Matlab / Simulink, were done. The results obtained from the experimental data returned a maximum position error of $4,48 \mathrm{~mm}$ for positive steps inputs, which was considered satisfactory for industrial applications. The comparison between simulated and experimental responses shows that the mathematical model presents a satisfactory approximation from the real system, although the experimental results have a faster stabilization time than the simulation, the transient response and the errors were similar.
\end{abstract}

control.

Keywords: positioning system, pneumatic actuator, proportional pressure regulators, PID control, pressure

\section{INTRODUCTION}

The use of pneumatic actuators presents some advantages when compared with other systems like hydraulic and electric, such as: economic operation; efficiency; durability; reliability; adaptability to hostile environments; safety for use near to flammable materials; are environmentally friendly; and have a low implementation cost [1]. However, there are nonlinearities that make difficult the position control of pneumatic actuators, such as: compressibility of the air; dead-zone; dynamic friction; and the nonlinear relation of the flow in the control holes [2]. Pneumatic systems have applications in different industries, for example: industrial automation; robotics; medical rehabilitation; automotive industries and mining plants.

Many studies about positioning systems using pneumatic actuators perform the proportional positioning through the control of the air mass flow driven to the actuator chambers, like presented in [1-3]. This type of operation limits the options of valves to the expensive proportional directional models. With the objective of cost reduction, some researches adopted cheaper on/off 2-way valves commanded by the Pulse Width Modulation (PWM) technique to the control of the air mass flow directed to the cylinder chambers. In this research, the positioning was made by the pressure control of the air directed to the front and rear chambers of the pneumatic cylinder. This configuration presents an alternative to the traditional proportional directional valves, allowing the designer to analyze the best cost $\mathrm{x}$ benefit to the project. The system was composed by two proportional pressure regulator valves that were set to work in double loop mode and each valve was equipped with one onboard PI controller. The PI tuning was done following the second technique proposed by Ziegler-Nichols. 


\section{SYSTEM DESCRIPTION}

The system was mainly composed by:

- 1 pneumatic cylinder, simple rod, double action (2" bore x 10" stroke) provided with an internal linear resistive transducer (linearity of $\pm 1 \%$ of full stroke and $\pm 2,54 \cdot 10^{-5} \mathrm{~m}$ mechanical repeatability). Model 20AM2-10A / Manufacturer Asco Numatics;

- 2 proportional pressure regulator valves. Model 609212611-Aoo / Manufacturer Asco Numatics (Hysteresis $<1 \%$ of the pressure range and Linearity / Repeatability $<0,5 \%$ of the pressure range);

- 1 communication module with protocol Ethernet-IP. Model 240-181 / Manufacturer Asco Numatics;

- 2 pressure transmitters. Model 2088G2S22A1M5Q4 / Manufacturer Rosemount (Precision 0,075\%);

- 1 power supply 24Vcc/5A. Model 2320908 / Manufacturer Phoenix Contact;

- 1 process controller. Model 199.20166 / Manufacturer Contemp;

- 1 process indicator. Model 199.20469 / Manufacturer Contemp;

- 1 signal splitter 4-20mA. Model 857-423 / Manufacturer Wago;

- 1 data acquisition (DAQ) board. Model USB 6008 / Manufacturer National Instruments.

In this system, the valves control the air pressure inside both front and rear chambers of the cylinder. The pressure transmitters monitor these pressures and send the data to the DAQ. The setpoint is in the form 4-20mA and can comes from the process controller (local setpoint) or from the communication module with industrial protocol (remote setpoint), the selection is made by the change-over switch. The same setpoint signal is sent to the both valves, but the valve responsible to control the pressure in rear chamber receives the signal in the form o to $100 \%$, while the other valve receives in the form 100 to $0 \%$, balancing the pressures and positioning the cylinder rod in the desired position. The feedback signal comes from the linear resistive transducer located inside the cylinder rod and it is distributed to the valves, process indicator and DAQ. The process indicator, in addition to the function of showing the current cylinder position, is also responsible to supply the transducer inside the cylinder rod with 10Vcc. The Figure 1 presents the prototype developed for tests.

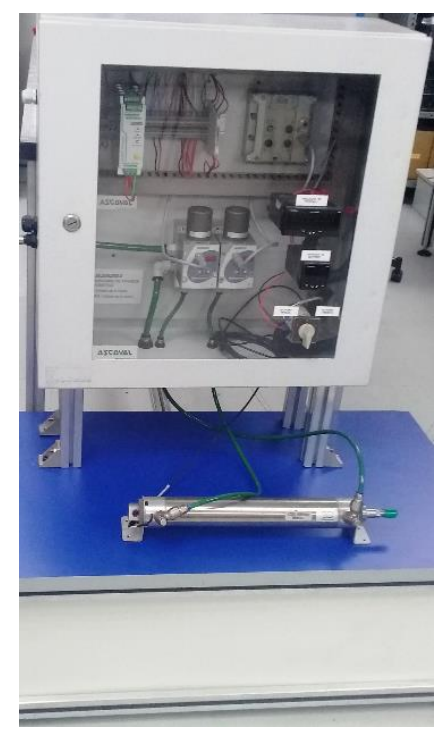

Figure 1 - Prototype

The Figure 2 presents a schematic diagram of the system. 


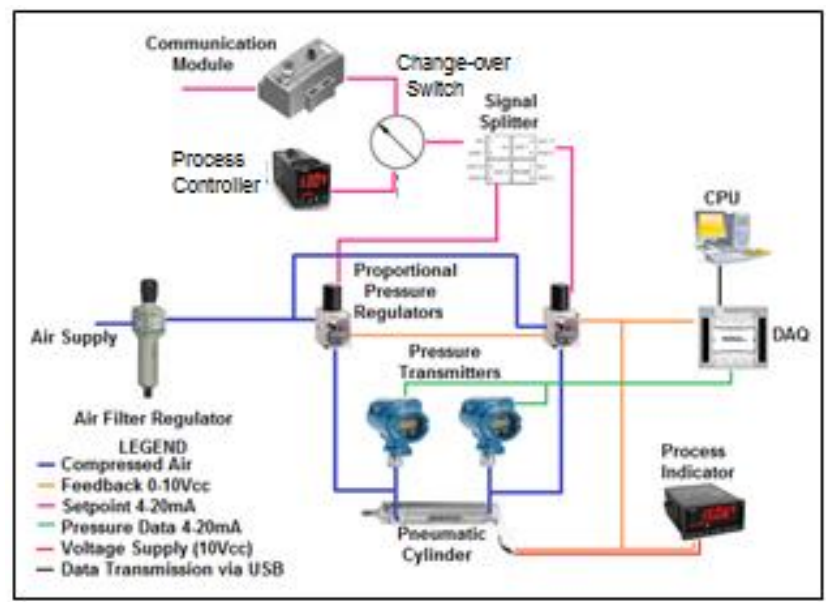

Figure 2 - Schematic Diagram of the System

A simplified block diagram of the system is showed in Figure 3.

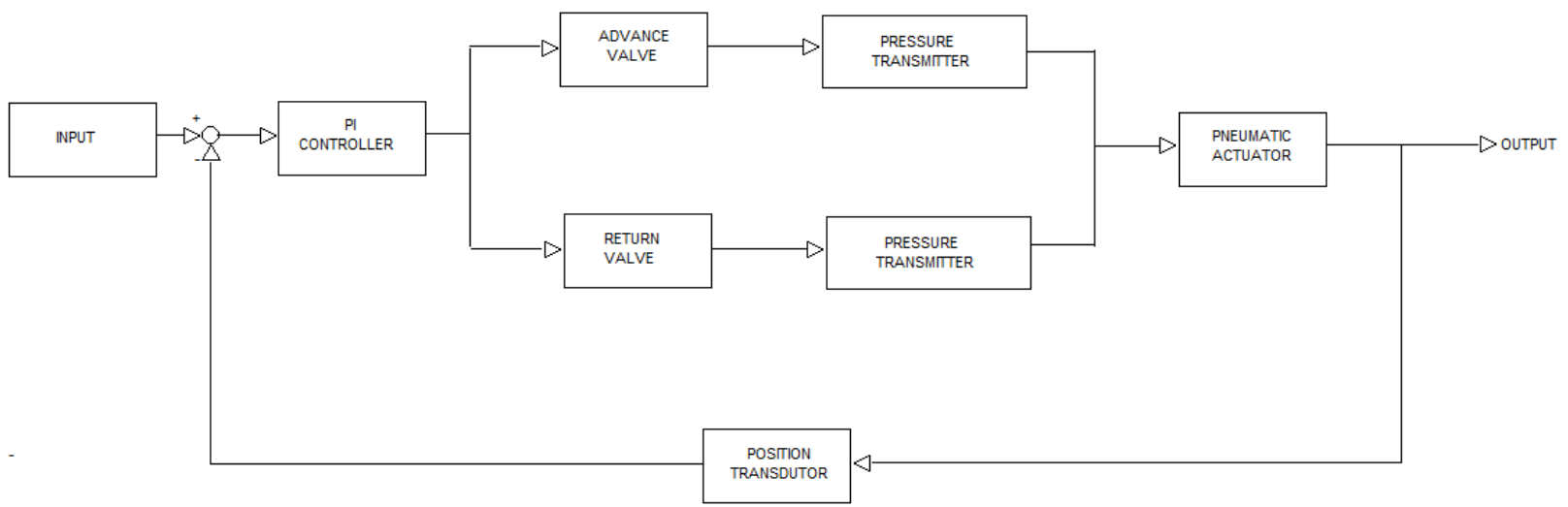

Figure 3 - Simplified block diagram of the system

In addition to the pressure transmitters installed in the pneumatic cylinder ports to allow the data collection, each valve has an internal pressure transmitter that allows the double loop configuration. This means that the valves have an internal pressure transmitter that sends the output pressure signal to the controller. Also, the PI Controller in the block diagram showed in the Figure 3 is an onboard controller located inside the proportional valves. The both controllers were tuned with the same parameters, so the block diagram is simplified with only one PI Controller to enable better understanding.

\section{MATHEMATICAL MODELLING}

The mathematical model of the system can be divided in two parts: Equation of Motion and Pressure Dynamics. The Equation of Motion describes the balance of forces, considering the friction compensation, and the Pressure Dynamics takes into account the air volume and the internal pressure variation, besides to present the relation between the front and rear chambers of the pneumatic cylinder.

The Equation of Motion and the Pressure Dynamics were defined based in the model suggested by [4]. Once the author adopted in his research a symmetrical cylinder, it was necessary to bring the model to the reality of the present research, where was adopted a non-symmetrical simple rod actuator that has different piston areas in the rear and front chambers. The Equation of Motion adopted in the present research is showed in Equation 1.

$$
\ddot{x}=\frac{1}{m}\left(A_{A} \cdot P_{A}-A_{B} \cdot P_{B}-F_{f}-\beta \dot{x}\right)
$$

Where $\ddot{x}$ is the piston acceleration, $\dot{\boldsymbol{x}}$ is the piston velocity, $\boldsymbol{m}$ is the load and piston mass $\left(\boldsymbol{m}=\boldsymbol{m}_{p}+\boldsymbol{m}_{L}\right), \boldsymbol{A}_{A}$ and $\boldsymbol{A}_{\boldsymbol{B}}$ are the piston area in the rear and front chambers respectively, $\boldsymbol{P}_{\boldsymbol{A}}$ and $\boldsymbol{P}_{\boldsymbol{B}}$ are the pressure inside the rear and front chambers respectively, $\boldsymbol{F}_{\boldsymbol{f}}$ is the friction force and $\boldsymbol{\beta}$ is the viscous friction coefficient. 
The Pressure Dynamics for the rear and front chambers are presented in the Equations 2 and 3.

$$
\begin{gathered}
\dot{P}_{A}=\frac{P_{0} \cdot K_{v}}{V_{t} / 2} \cdot \frac{U}{U_{n}}-\frac{P_{A} \cdot A_{A}}{V_{t} / 2} \cdot \dot{x} \\
\dot{P}_{B}=-\frac{P_{0} \cdot K_{v}}{V_{t} / 2} \cdot \frac{U}{U_{n}}+\frac{P_{B} \cdot A_{B}}{V_{t} / 2} \cdot \dot{x}
\end{gathered}
$$

Where $\boldsymbol{P}_{\mathbf{0}}$ is the ambient pressure, $\boldsymbol{K}_{\boldsymbol{v}}$ is the valve flow coefficient, $\boldsymbol{V}_{\boldsymbol{t}}$ is the total air volume, $\boldsymbol{U}$ is the current position signal and $\boldsymbol{U}_{\boldsymbol{n}}$ is the maximum position signal.

The friction compensation adopted was based in the model suggested by [3] and takes into account the viscous friction coefficient $\beta$ and the Static-Coulomb friction force $F_{f}$, where $F_{f}$ is defined by:

$$
F_{f}= \begin{cases}F_{s f} \operatorname{sign}(F p) & \text { if } \dot{x}=0 \\ F_{d f} \operatorname{sign}(\dot{x}) & \text { if } \dot{x} \neq 0\end{cases}
$$

Where $\boldsymbol{F p}$ is the pneumatic force $\left(\boldsymbol{F p}=\boldsymbol{A}_{\boldsymbol{A}} \cdot \boldsymbol{P}_{\boldsymbol{A}}-\boldsymbol{A}_{\boldsymbol{B}} \cdot \boldsymbol{P}_{\boldsymbol{B}}\right), \boldsymbol{F}_{\boldsymbol{s} f}$ is the static friction force, $\boldsymbol{F}_{\boldsymbol{d} f}$ is the Coulomb friction force and $\operatorname{sign}(\dot{\boldsymbol{x}})$ is given by:

$$
\operatorname{sign}(\dot{x})=\left\{\begin{aligned}
-1 & \text { if } \dot{x} \leq-1 \\
0 & \text { if } \dot{x}=0 \\
1 & \text { if } \dot{x} \geq 1
\end{aligned}\right.
$$

The Figure 4 presents a schematic representation of the system.

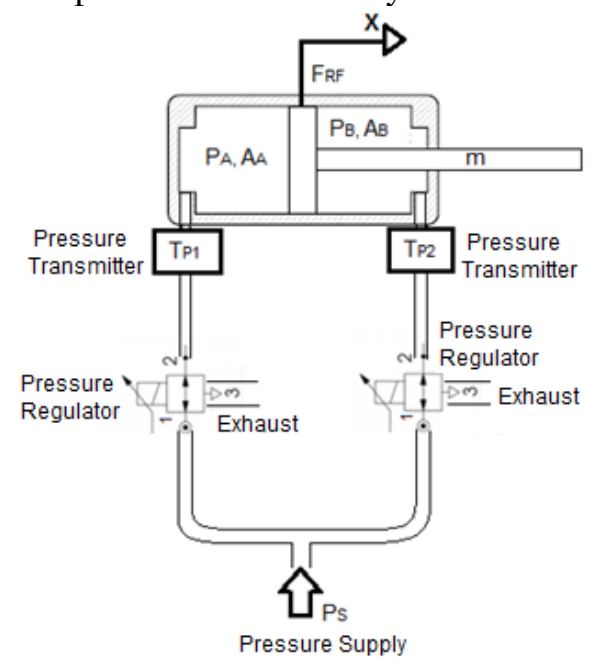

Figure 4 - Schematic Representation of the System

The Table 1 shows the real values adopted for the parameters.

Table 1 - Parameters of the cylinder

\begin{tabular}{c|c|c}
\hline \multicolumn{2}{c|}{ Parameter } & Value \\
\hline $\begin{array}{c}\text { Effective piston area } \\
\text { (advance) }\end{array}$ & $A_{A}$ & $\begin{array}{c}18.67 \times 10^{-4} \\
{\left[\mathrm{~m}^{2}\right]}\end{array}$ \\
$\begin{array}{c}\text { Effective piston area } \\
\text { (return) }\end{array}$ & $A_{B}$ & $16.8 \times 10^{-4}\left[\mathrm{~m}^{2}\right]$ \\
Total air volume & $V_{t}$ & $4.47 \times 10^{-4}\left[\mathrm{~m}^{3}\right]$ \\
\hline
\end{tabular}




\begin{tabular}{|c|c|c|}
\hline $\begin{array}{l}\text { Viscous friction } \\
\text { coefficient }\end{array}$ & $\beta$ & $4.47[\mathrm{~N} /(\mathrm{m} / \mathrm{s})]$ \\
\hline $\begin{array}{l}\text { Absolut ambient } \\
\text { pressure }\end{array}$ & $P_{0}$ & $1 \times 10^{5}[\mathrm{~Pa}]$ \\
\hline Valve flow coefficient & $K_{v}$ & $\begin{array}{c}3.33 \times 10^{-4} \\
{\left[\mathrm{~m}^{3} / \mathrm{s}\right]}\end{array}$ \\
\hline $\begin{array}{l}\text { Mass of the assembly } \\
\text { piston + rod }\end{array}$ & $m$ & $0.49[\mathrm{Kg}]$ \\
\hline Dynamic friction force & $F_{d f}$ & $0.49[\mathrm{~N}]$ \\
\hline Static friction force & $F_{s f}$ & $3.82[\mathrm{~N}]$ \\
\hline Pressure supply & $P_{S}$ & $6 \times 10^{5}[\mathrm{~Pa}]$ \\
\hline
\end{tabular}

\section{RESULTS}

The PI tuning was done following the second technique presented by Ziegler-Nichols, where a proportional gain is raised until the system reaches periodic oscillations, then it is possible to measure the critic period and apply the results in the equations. The PI parameters were defined as $K_{p}=6,3$ and $T_{i}=1,325$. The results comparison showed that, despite the simulated curve was not identical to the experimental result, the model has given a satisfactory approximation to the real response. Although the real system presented a stabilization time of $4,5 \mathrm{~s}$ and the simulation presented a stabilization time of $16 \mathrm{~s}$, the transient response and errors were similar. The maximum error obtained in the permanent regime of the experimental result was $4,48 \mathrm{~mm}$ for positive steps inputs and 3,61mm for negative steps inputs. Figure 5.

The comparison between the experimental and simulated results with a positive step input is showed in the

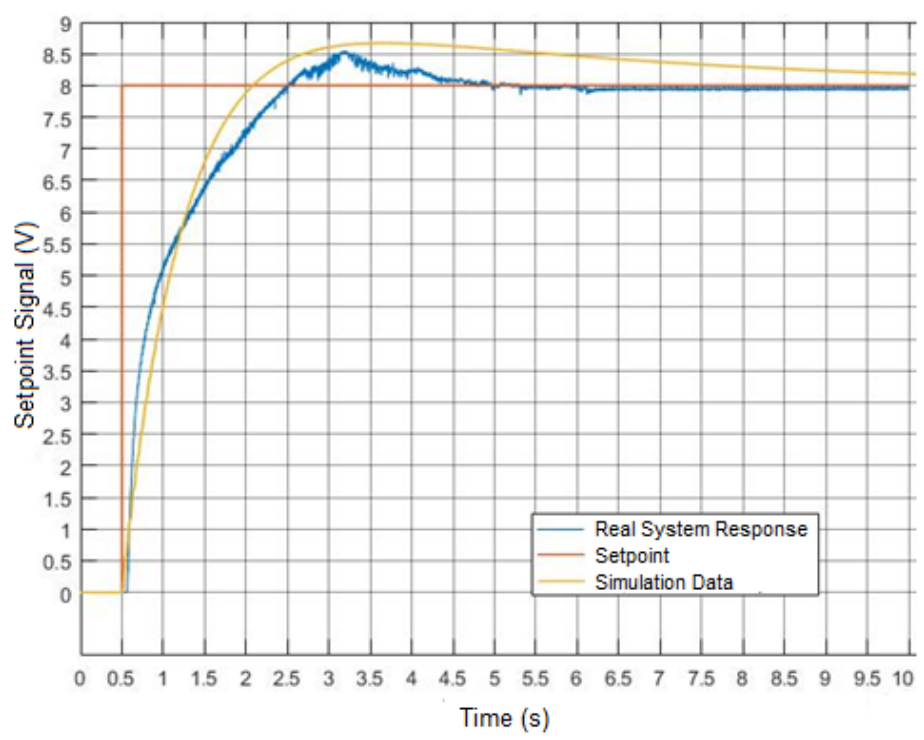

Figure 5 - Results comparison for a positive step input

\section{CONCLUSION}

The results analysis permits to conclude that the proposed model gives a satisfactory approximation to the real results and allows the realization of studies with different applications and parameters. The experimental results were considered satisfactory for the use of the system in industrial applications. 
Other results and analysis, besides more information regarding the mathematical model development and computational simulation are described in [5].

Special thanks to the Emerson Industrial Automation Brazil, Fluid and Motion Control, for all the support dispensed for this research. Without this support, it would not be possible to conclude this study.

\section{REFERENCES}

[1] DULAU, M., NAGY, G., TATAR, A. Experiments of pneumatic linear positioning with conventional control system. Tirgu Mures: Scientific bulletin of the Petru Maior University of Tirgu Mures, Tirgu Mures, v.12, n.1, pp. 5-9, 2015.

[2] LIU, Y.-T., KUNG, T.-T., CHANG, K.-M., CHEN, S.-Y. Observer-based adaptive sliding mode control for pneumatic servo system. Elsevier, Kaohsiung, 30 Jul. 2012, pp. 522-525, disponível em: www.elsevier.com/locate/precision. Acesso em: 25 Dez. 2015.

[3] RICHER, E., HURMUZLU, Y. A high performance pneumatic force actuator system: Part 1 - Nonlinear Mathematical Model. ASME Journal of dynamic systems measurement and control, Dallas, 12 Fev. 2001, v.122, n.3, pp. 416-425.

[4] SITUM, Z. Control of a pneumatic drive using electronic pressure valves. Transations of the Institute of Measurement and Control, Zagreb, 2013, pp.1085-1093.

[5] MUZY, G. Positioning system of a pneumatic actuator commanded by proportional pressure regulator valves. São Paulo, SP: 2018. Masters Dissertation (Master's degree in automation and process control) - Federal Institute of Education, Science and Technology of São Paulo. 